\title{
Numerical Simulation of Simultaneous Growth of Multiple Interacting Hydraulic Fractures from Horizontal Wells
}

Andrew P. Bunger ${ }^{1}$, Ph.D., and Anthony P. Peirce ${ }^{2}$, Ph.D.

\footnotetext{
${ }^{1}$ Assistant Professor, Department of Civil and Environmental Engineering, Swanson School of Engineering, University of Pittsburgh, 3700 O’Hara Street, Pittsburgh, PA 15261, USA, email: bunger@pitt.edu

${ }^{2}$ Professor, Department of Mathematics, The University of British Columbia, \#121-1984 Mathematics Road, Vancouver, B.C. V6T 1Z2, Canada, email: peirce@math.ubc.ca
}

\begin{abstract}
The technique of multistage hydraulic fracturing from horizontal wells is universally credited with enabling the economical production of hydrocarbon resources from shale formations. The method almost always entails the injection of fluid through the wellbore with the potential to create hydraulic fractures from multiple reservoir entry points, typically clusters of wellbore perforations, that are spaced out along the wellbore within a section that is colloquially referred to as a "stage". Arguably the most basic question about this situation is how many perforation clusters within a given fracturing stage can be expected to produce growing hydraulic fractures. This paper presents a numerical investigation of this issue that employs a newly-developed, fully coupled parallel planar 3D hydraulic fracturing simulator that features: implicit time stepping, an implicit level set scheme to locate the propagating hydraulic fracture fronts that respond to their regimes of propagation and enables highly accurate simulations using a very coarse mesh, and the capability to dynamically partition the fluid among multiple, simultaneously growing hydraulic fractures in parallel, overlapping planes. Our results demonstrate the dependence of the energetically preferred number of growing hydraulic fractures on the length of the isolated zone, the height of the reservoir, and the relative importance of the fluid viscosity. In particular, we show that reservoirs with effective height containment and injection strategies that ensure substantial viscous dissipation will promote growth of multiple simultaneous hydraulic fractures rather than localization to just one or two dominant fractures.
\end{abstract}

\section{INTRODUCTION}

Multistage hydraulic fracturing from horizontal wells is currently the petroleum industry's most important reservoir stimulation technology; without it hydrocarbons from shale reservoirs could not be produced economically (e.g. King 2010). However, production logging often shows that more than half of perforation clusters are non-producing (Miller and Waters 2011).

This failure to generate uniformly distributed production highlights two issues that are currently detrimental to optimal recovery. The first is failure to adapt the well 
completion strategy in light of variability of reservoir properties, including the in-situ stress, along the well (e.g. Baihly et al. 2011). The second is the propensity of hydraulic fractures (HFs) to exert stresses on each other thereby suppressing the growth of some or most of the HFs attempting to extend from an array of entry points such as perforation clusters. This phenomenon, called "stress shadowing", has been studied using multiple fracture models for well over a decade (e.g. Gemanovich et al. 1997, Olson 2004, Fisher et al. 2004, Abass et al. 2009, Meyer and Bazan 2011).

These two issues, and their management, are at the core of mechanicallyjustified engineering decisions - such as length of each stimulation interval and spatial distribution of perforation clusters within the interval - which will have a first order effect on the productivity of the well. It is therefore vital to improve on the current situation wherein these critical engineering decisions are often made with little guidance from models that rigorously capture the essential physical mechanisms that determine the impacts of these decisions on HF growth.

Recognizing the value of simulations that capture the complexity that can be generated through the interaction of multiple, simultaneously growing hydraulic fractures (e.g. Germanovich et al. 1997, Olson and Dahi-Taleghani 2009, Damjanac et al. 2010, Cipolla et al. 2011, Nagel et al. 2011, Meyer and Bazan 2011, Kresse et al. 2013, McClure and Horne 2013), a different and complimentary approach has recently been developed that uses simple analytical models to clarify the basic contributors to the energy balance of a system of multiple, simultaneously growing and parallel HFs (Bunger 2013). The analysis is built on the premise is that, given a well interval of a certain length with multiple entry points, the number of HFs that will actually grow corresponds to the number that minimizes the required energy input. The resulting analytical models show how the required energy input depends on the number of HFs and how this dependence is affected by HF geometry, fluid viscosity and injection rate, mechanical properties of the rock, and perforation geometry (Bunger 2013, Bunger et al. In Press).

The analytical modeling has therefore proven useful for discerning the mechanisms that control the overall behavior of the system. But the approach is restrictive on both the geometry and the number of coupled phenomena that can be considered. Hence there is a need for a new, fully-coupled numerical simulator that can systematically test the analytical predictions and that can be progressively enhanced to capture increasingly more complex behavior. Such a model can therefore be seen as providing bridge between theory and the predictions of design-oriented HF simulators that prioritize rapid computation enabled by substantial simplifications of the underlying mechanical model, which almost always include a local treatment of the elasticity equations according to the so-called pseudo 3D approach (e.g. Meyer and Bazan 2011, Kresse et al. 2013).

As a first step in this reconciliation between theory and numerical simulation, this paper presents a comparison between the analytical predictions of Bunger (2013) and a newly-developed, fully coupled parallel planar 3D hydraulic fracturing simulator. This new simulator features an implicit level set scheme to locate the propagating hydraulic fracture fronts that respond to their regimes of propagation and enables highly accurate simulations using a very coarse mesh, and the capability to dynamically partition the fluid among multiple, simultaneously growing hydraulic 
fractures in parallel, overlapping planes. Consistent with the predictions of Bunger (2013), these simulations demonstrate that two factors, substantial dissipation of energy through viscous fluid flow and effective containment of height growth, are critical to promoting simultaneous growth of multiple hydraulic fractures.

\section{SUMMARY OF ANALYTICAL PREDICTIONS}

Bunger (2013) and Bunger et al. (In Press) discovered that there are 3 critical factors that determine the propensity for multiple hydraulic fractures to growth simultaneously. These are: 1) HF geometry, 2) perforation pressure loss, and 3) dissipation of energy through viscous fluid flow. Hence we can contrast arrays of plane strain (2D), radial (penny-shaped), and blade-like (or "PKN") HFs (see Fig. 1), each of which gives strikingly different predicted behavior. For a start, the 2D cases always favor growth of one HF regardless of the number of and/or spacing between the entry points. Because this geometry is common for modelling but not very representative even of idealized HFs in the field, we conclude that 2D simulators will overstate the tendency of HFs to localize.
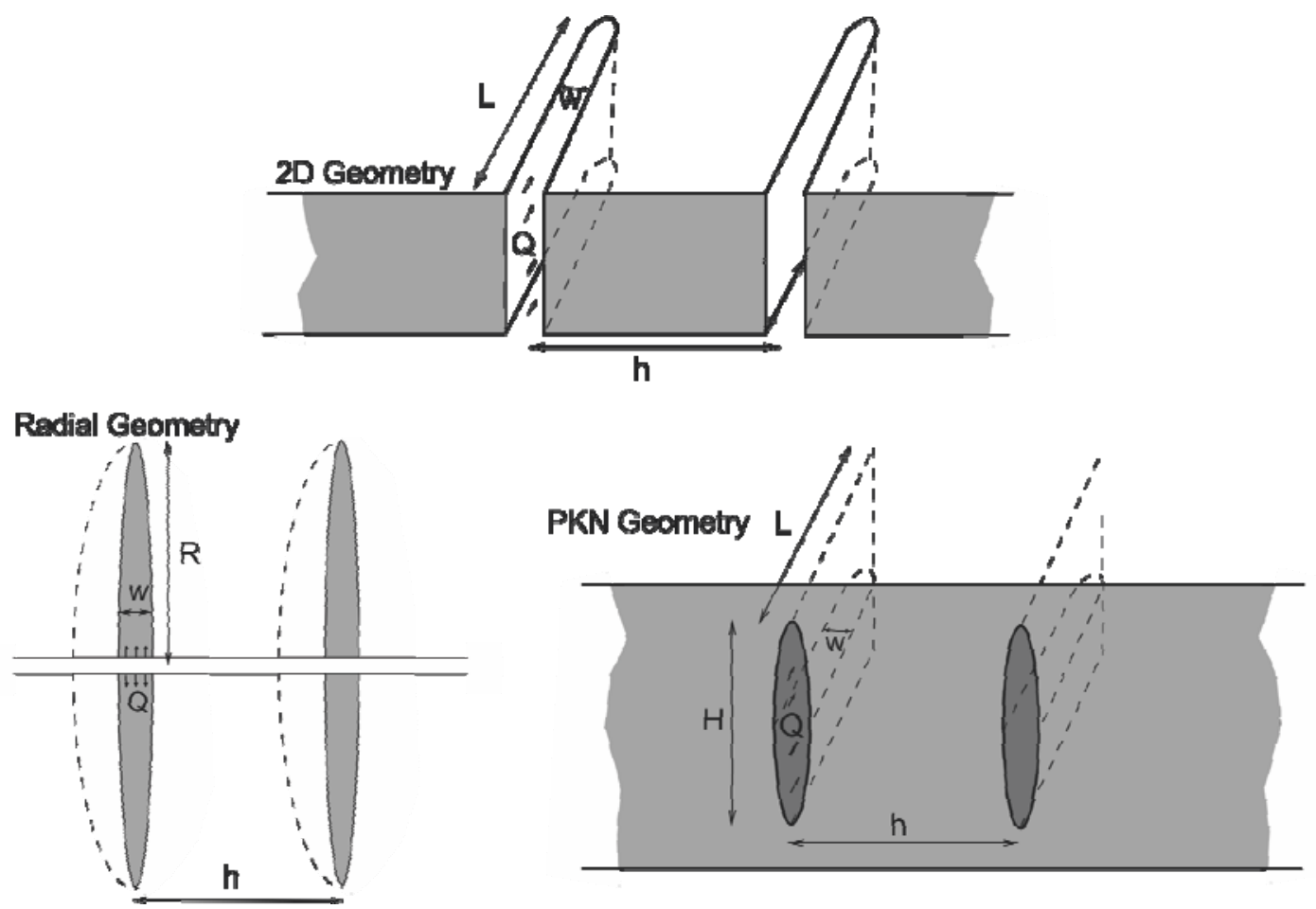

FIG. 1. HF geometries, re-drawn based on Bunger (2013).

In contrast, arrays of penny-shaped hydraulic fractures favor growth from all perforation clusters as long as viscous dissipation of energy dominates the energy dissipation associated with breakage of the rock. Radial fractures will persist until their radius is around 0.8 times the spacing, at which point the elastic interaction or "stress shadow" effect causes a progressive localization to fewer HFs. If rock fracture dominates the energy balance, penny-shaped HF arrays will immediately favor growth of a single HF. "Limited entry" techniques (e.g. Howard and Fast 1970, 
Economides and Nolte 2000) that deliberately increase the energy dissipation at the entry points can mitigate this localization until the radius is around 0.2 times the spacing. We therefore conclude that factors promoting viscosity-dominated growth (large injection rate, large viscosity, stiff rock, and low toughness rock) contribute to growth from as many perforation clusters as possible when the HFs are roughly penny-shaped.

For height limited HFs that attain a PKN geometry the behavior is different again. Here the model predicts an energetically preferred spacing that results from a competition. On the one hand, minimization of the viscous energy dissipation drives the system to favor very closely spaced HFs, as was shown for the penny-shaped case. On the other hand, the stress shadow energetically penalizes the system when the HFs are close together. In the PKN case these competing processes result in an energetically-preferred spacing that is around 2.5 times the HF height in the absence of perforation losses and can be as little as 1.2 times the HF height with large perforation losses. This prediction is supported by previously observed patterns of microseismicity in the Barnett Shale (Fisher et al. 2004 as discussed by Bunger et al. In Press).

\section{DESCRIPTION OF THE NUMERICAL MODEL}

The numerical model assumes that fractures are propagating within parallel planes that are spaced $h_{k}$ apart in a perforation stage of length $Z$ within a pay zone of height $H$ (see Fig. 2). The numerical model uses the Implicit Level Set Algorithm (ILSA) (Peirce and Detournay, 2008) to model the propagation of fractures with arbitrarily shaped boundaries within each of the parallel planes, which are assumed to be perpendicular to the minimum principal stress direction. The fractures are assumed to propagate in a three dimensional elastic medium at a rate that is sufficiently slow for the elastic medium to be in a state of static equilibrium.

The elastic equilibrium equations are discretized using the displacement discontinuity boundary integral method in which the fracture within each plane is represented by constant width rectangular elements that are collocated at element centers. The Reynolds lubrication equation, expressing the conservation of mass of the viscous fluid contained within the crack surfaces of each of the fractures, is discretized using a finite volume method, which is also defined with respect to quantities sampled at the centers of the rectangular elements. At the periphery of the fractures, which may not conform to the structured rectangular mesh, the fracture boundaries are represented using a concept of partially filled tip elements that are used to define average fracture widths, which are also sampled at element centers.

The distinguishing feature of this algorithm is its ability to locate the free boundaries of the fractures using the asymptotic behavior of the hydraulic fracture widths that are applicable at points in the neighborhood of the perimeters of the fractures. For the $k^{\text {th }}$ fracture the free boundary is located by the following iterative process: given initial guesses for each of the fracture boundaries $\partial S_{k}$, determine the corresponding equilibrating and volume conserving fracture widths $w_{k}$ and fluid pressures $p_{f, k}$, and well-bore fluxes $q_{k}$ subject to the constraints that the well-bore fluxes sum to the total fluid volume pumped and the well-bore pressures are the same across the array. In the ribbons of elements that are completely filled with fluid and, 
which share at least one side with a partially filled tip element, use the trial width values to estimate the distance to the free boundary by inverting the applicable tip asymptotic behavior (Detournay, 2004); use these estimates of the distances to the free boundaries as initial conditions for the eikonal equations $\left|\nabla T_{k}(x, y)\right|=1$, whose level set curves $T_{k}(x, y)=0$ define the free boundaries. The fracture boundaries are then moved to the curves $T_{k}(x, y)=0$ and the iterative process is repeated until convergence is achieved. The algorithm uses the multi-scale hydraulic fracture tip asymptotic solution (Detournay, 2004) and thus automatically captures the different types of propagation regimes with relatively coarse meshing of the fracture planes. This iterative procedure is performed for each of the fractures in each of the fracture planes. The algorithm is described in more detail in Peirce and Bunger (In Review).

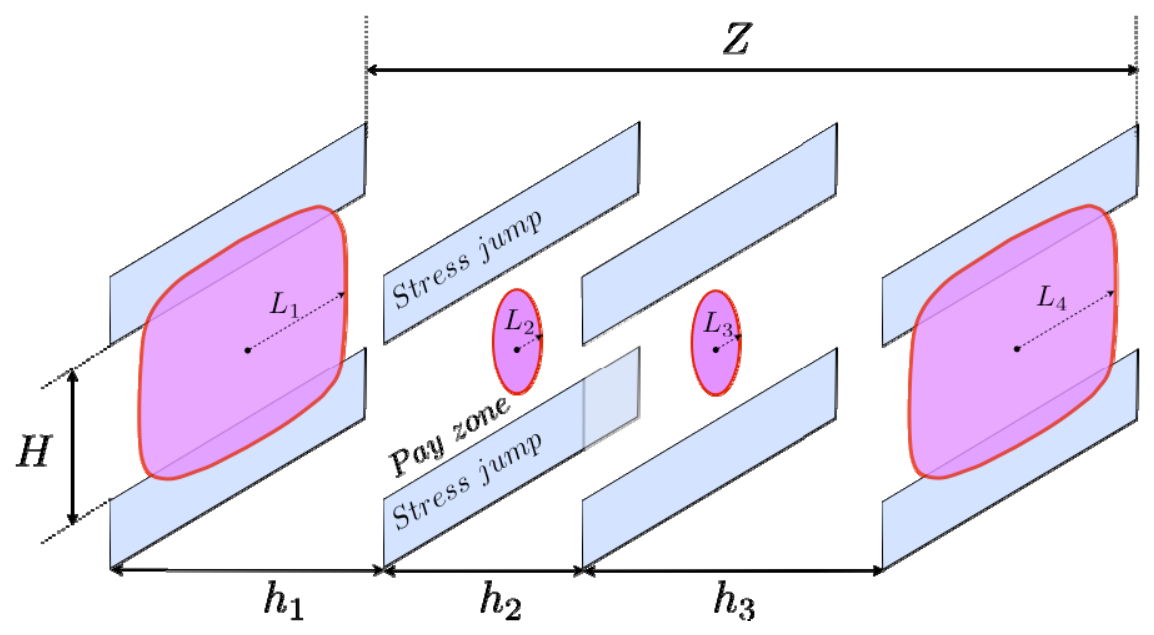

FIG. 2. Geometric configuration of the experimental perforation stage of length $Z$ within a pay zone of height $H$ in which $h_{k}$ is the fracture spacing. After Peirce and Bunger (In Review).

\section{NUMERICAL RESULTS}

\section{Radial Growth in Toughness Dominated Regime}

Firstly we simulate simultaneous injection into 5 entry points spaced uniformly at 30 $\mathrm{m}$ so as to cover a $120 \mathrm{~m}$ interval of the wellbore. Injection parameters are chosen so that the total simulation time satisfies (e.g. Detournay 2004)

$$
t_{T O T}>\left(\frac{\mu^{\prime} Q_{o}^{3} E^{\prime}}{K^{\prime 18}}\right)^{1 / 2}
$$

where $Q_{o}$ is the volumetric injection rate and 1) $\mu^{\prime}=12 \mu$ for the fluid viscosity $\mu$, 2) $E^{\prime}=E /\left(1-v^{2}\right)$ for the Young's modulus $E$ and Poisson's ratio $v$, and 3$)$ $K^{\prime}=(32 / \pi)^{1 / 2} K_{I c}$ for the rock fracture toughness $K_{I c}$. This constraint provides a convenient definition of the so-called toughness dominated regime for radial HFs, which can be considered the case wherein the energy dissipated due to rock breakage far exceeds the energy that is dissipated through viscous fluid flow (e.g. Lecampion and Detournay 2007). Consistent with this constraint, the following parameter values 
are chosen for this example:

$$
\begin{array}{ll}
E=9.5 \mathrm{GPa} & v=0.2 \quad K_{I c}=1.5 \mathrm{MPa} . \mathrm{m}^{1 / 2} \\
\mu=0.001 \mathrm{~Pa} . \mathrm{s} & Q_{0}=0.0265 \mathrm{~m}^{3} / \mathrm{s} \quad Z=120 \mathrm{~m} \\
\sigma_{z z}^{0}=70 \mathrm{MPa} & \Delta \sigma_{z z}^{0}=0 \mathrm{MPa}
\end{array}
$$

Fig. 3a shows a snapshot of the 5 HFs when the outer 2 have reached a radius of about $30 \mathrm{~m}$. The inner HFs are clearly suppressed. Fig. 3b shows that the inner HFs grow uniformly with the outer HFs until their radius is about 0.15 times they spacing. Localization then commences favoring the outer HFs while the inner HFs attain a radius around 0.2 times the spacing and do not grow any further over the duration of the simulation.

\section{Radial Growth in Viscosity Dominated Regime}

As in the previous case, here we simulate simultaneous injection into 5 entry points spaced uniformly at $30 \mathrm{~m}$ so as to cover a $120 \mathrm{~m}$ interval of the wellbore. However, here the injection parameters are chosen so that the total simulation time satisfies (e.g. Detournay 2004)

$$
t_{T O T}<<\left(\frac{\mu^{\prime} Q_{o}^{3} E^{\prime}}{K^{\prime 18}}\right)^{1 / 2}
$$

This constraint provides a convenient definition of the so-called viscosity dominated regime for radial HFs, which can be considered to be the case in which the energy dissipated due to rock breakage is far exceeded by the energy that is dissipated through viscous fluid flow (e.g. Lecampion and Detournay 2007). Within this constraint, the following parameters are chosen for this example:

$$
\begin{array}{lcc}
E=9.5 \mathrm{GPa} & v=0.2 \quad K_{I c}=0 \mathrm{MPa} . \mathrm{m}^{1 / 2} \\
\mu=1 P a . s & Q_{0}=0.1 \mathrm{~m}^{3} / \mathrm{s} \quad Z=120 \mathrm{~m} \\
\sigma_{z z}^{0}=70 \mathrm{MPa} & \Delta \sigma_{z z}^{0}=0 \mathrm{MPa}
\end{array}
$$

Fig. 4a shows a snapshot of the 5 HFs when the outer 2 have reached a radius of about $30 \mathrm{~m}$. The inner HFs somewhat suppressed in their growth, although not nearly as strikingly as in the toughness dominated case. In fact, as shown in Fig. 4b, even after the outer HFs begin to be favored when the radius attains about 0.3 times the spacing, the inner HFs continue to grow and only trail the outer HFs by about $20 \%$ when the radius of the outer HFs is approximately equal to the spacing. Hence we observe that for viscosity dominated HFs the localization commences later than in the toughness dominated regime (here "later" means that the radius attains a larger value relative to the spacing). Furthermore the localization is less pronounced once it commences so that the array grows in a much more uniform manner than it does in the toughness dominated regime. 

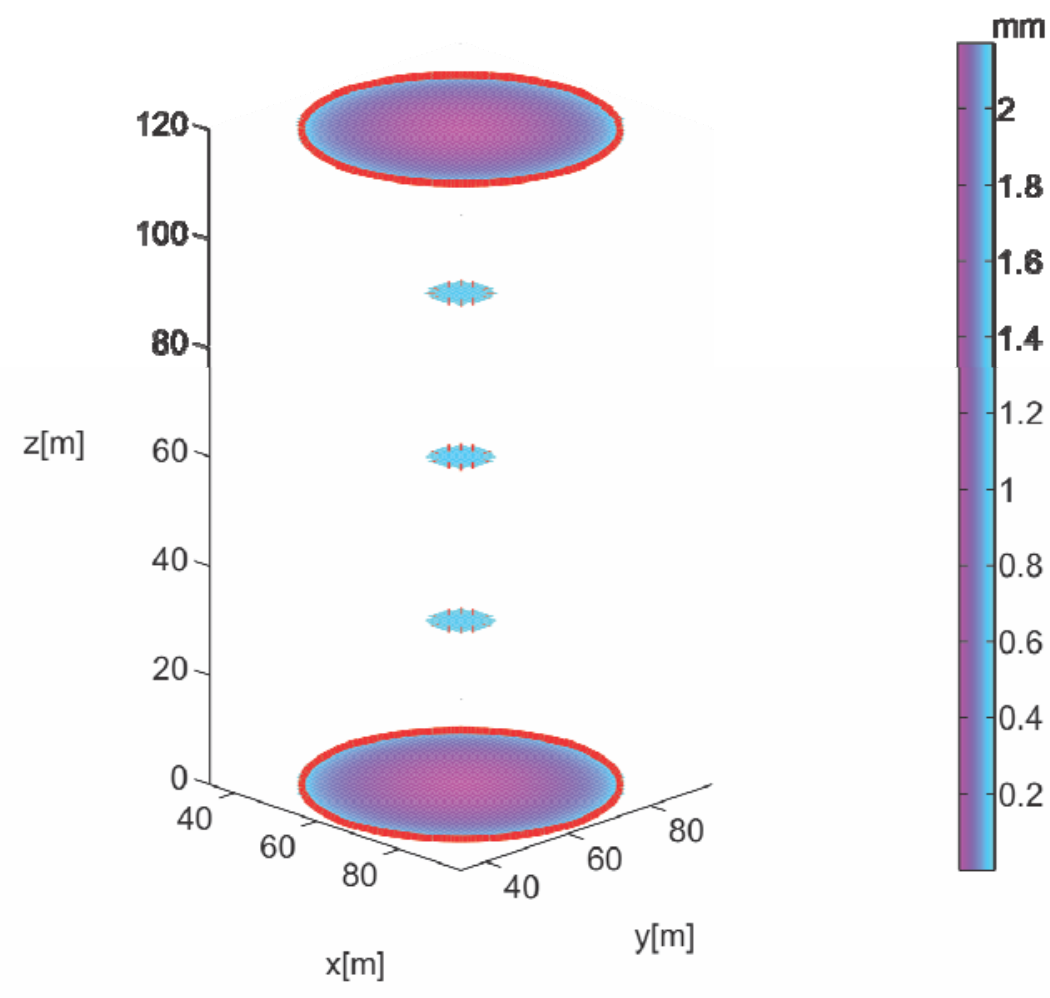

a)

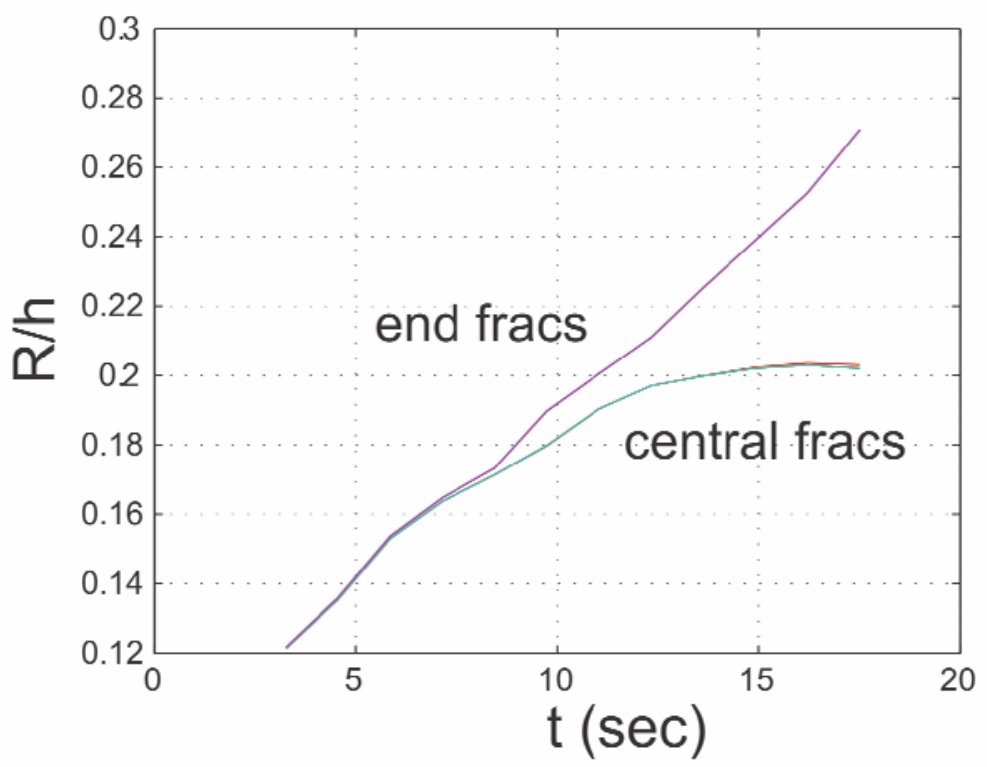

b)

FIG. 3. Radial toughness regime. a) 5 hydraulic fractures with contours corresponding to opening. b) Evolution of the radius of the fractures normalized by the spacing between them. 


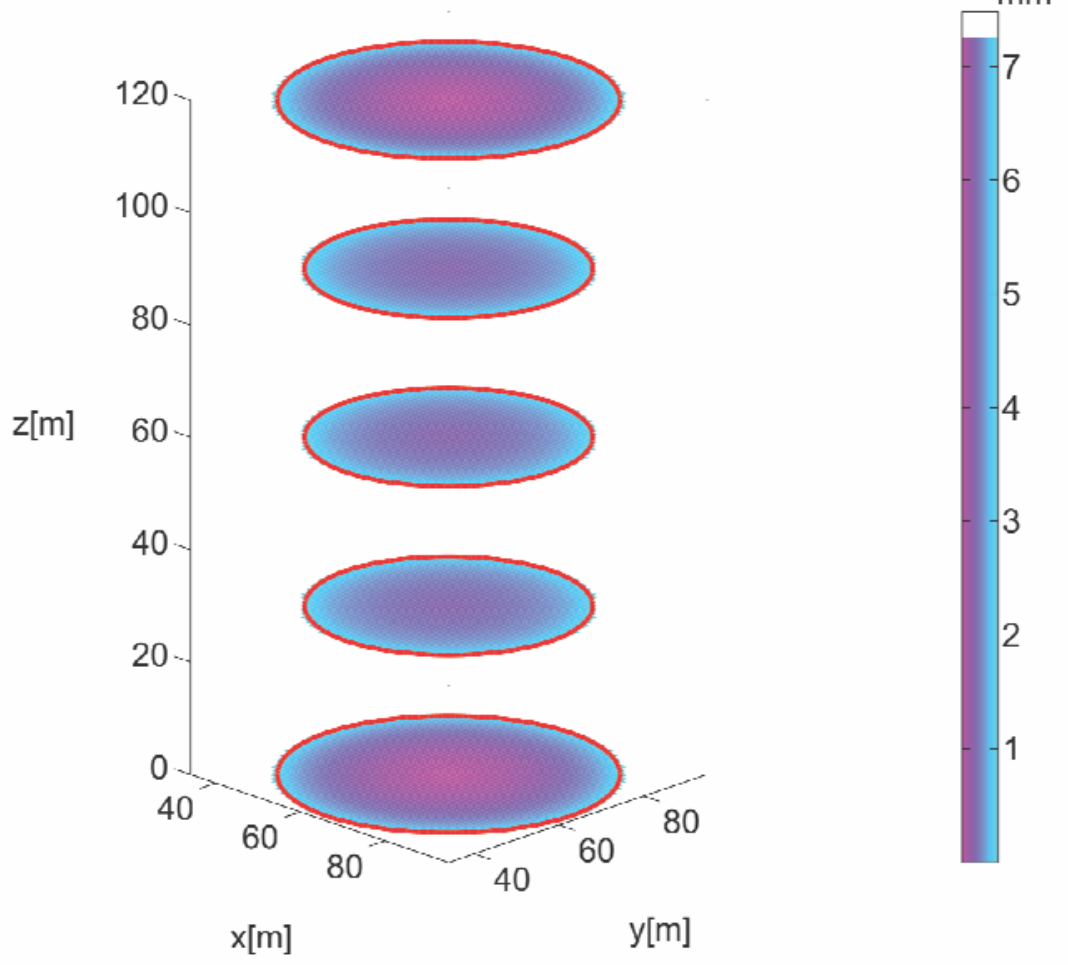

a)

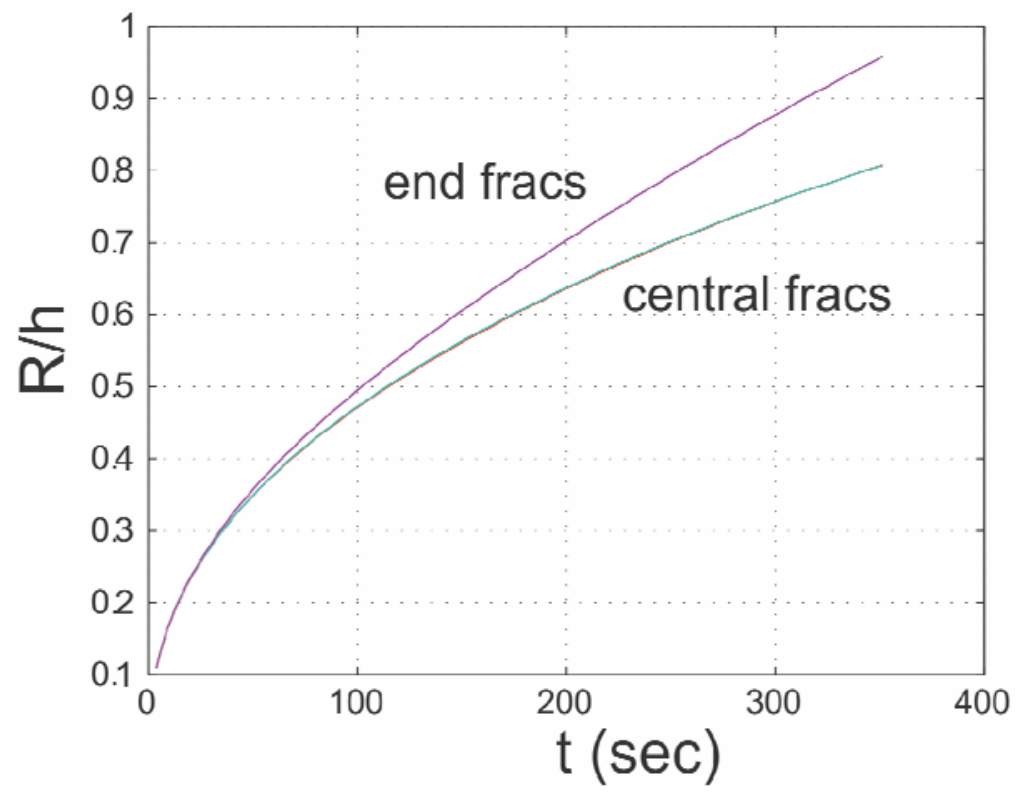

b)

FIG. 4. Radial viscosity regime. a) 5 hydraulic fractures with contours corresponding to opening. b) Evolution of the radius of the fractures normalized by the spacing between them. 


\section{Blade-like Growth with Stage Length Greatly Exceeding HF Height}

Here we simulate simultaneous injection into 7 entry points spaced uniformly at $20 \mathrm{~m}$ so as to cover a $120 \mathrm{~m}$ interval of the wellbore. Here we ensure growth that is strictly in the viscosity dominated regime by setting the rock fracture toughness to be small and the viscosity and pump rate to be relatively large. The set of parameters for this example are otherwise chosen as:

$$
\begin{array}{lcc}
E=9.5 \mathrm{GPa} & v=0.2 \quad K_{I c}=0.157 \mathrm{MPa} \mathrm{m}^{1 / 2} \\
\mu=1 P a . s & Q_{0}=0.0265 \mathrm{~m}^{3} / \mathrm{s} & Z=120 \mathrm{~m} \\
\sigma_{z z}^{0}=70 \mathrm{MPa} & \Delta \sigma_{z z}^{0}=5 \mathrm{MPa} & H=12 \mathrm{~m}
\end{array}
$$

The main difference between this case and the radial viscosity-dominated case is there here we have imposed the condition that the injection takes place within a 12 $\mathrm{m}$ wide strip of low stress so that height growth is naturally limited (although finite in contrast to the original models of Perkins and Kern 1961 and Nordgren 1972).

Fig. 5a shows a snapshot of the $7 \mathrm{HFs}$ when they have reached a length of about $40 \mathrm{~m}$. In this case the array is nearly uniform; the inner HFs trail behind in terms of length by only a few percent as shown in Fig. 5b. We observe, then, that height constraint promotes extensive uniform growth when the spacing is on the order of 2 times the HF height.

\section{Blade-like Growth with Stage Length Equal to HF Height}

The previous case showed the propensity of height limited HFs to extensively grow simultaneously in the viscosity dominated regime as long as their separation is large enough relative to their height. In contrast, we finally simulate $5 \mathrm{HFs}$ with a uniform spacing of $5 \mathrm{~m}$ so as to cover a $20 \mathrm{~m}$ interval within a $20 \mathrm{~m}$ high zone of low stress that limits height to approximately the same value. We ensure growth that is strictly in the viscosity dominated regime by setting the rock fracture toughness to zero. The set of parameters for this example are otherwise chosen as:

$$
\begin{aligned}
& E=9.5 \mathrm{GPa}, \quad v=0.2, \quad K_{I c}=0 \mathrm{MPa} \cdot \mathrm{m}^{1 / 2} \\
& \mu=1 \mathrm{~Pa} \cdot \mathrm{s}, \quad Q_{0}=0.1 \mathrm{~m}^{3} / \mathrm{s}, \quad Z=20 \mathrm{~m} \\
& \sigma_{z z}^{0}=70 \mathrm{MPa}, \quad \Delta \sigma_{z z}^{0}=5 \mathrm{MPa}, \quad H=20 \mathrm{~m}
\end{aligned}
$$

Fig. 6a shows the suppression of the inner HFs, consistent with a number of similar simulation results (e.g. Gemanovich et al. 1997, Olson 2004, Fisher et al. 2004, Abass et al. 2009, Meyer and Bazan 2011). However, somewhat surprisingly the suppression of the inner HFs can be mitigated, as shown in detail by Peirce and Bunger (In Review). Using the same pumped volume as that in the uniformly spaced array shown in Fig. 6a but with the entry points placed at $\mathrm{z}=0,3.5,10,16.5$, and $20 \mathrm{~m}$ instead, we obtain a completely different HF growth pattern as shown in Fig. 6b. Hence we find that specific non-uniformly spaced arrays can promote multiple simultaneous HF growth. In this example, as pointed out by Peirce and Bunger (In Review), the fracture surface area shown in Fig. 6b exceeds the fracture surface area in Fig. 6a by about 50\%. If primary hydrocarbon recovery scales with surface area, 
then recovery rates could be improved by as much as $50 \%$ through modification of the entry-point locations.

\section{DISCUSSION}

The overall contrasting behaviors are: 1 ) growth of only the outer HFs in the array for toughness dominated radial hydraulic fractures and for uniformly-spaced arrays of PKN hydraulic fractures, 2) growth of all HFs in an array for radial growth in the viscosity dominated regime until the radius is 0.5-1 times the spacing, 3) persistent growth of multiple HFs for arrays of PKN fractures that are either spaced more widely than their height or that are strategically non-uniformly spaced according to the method of interference fracturing proposed by Peirce and Bunger (In Review).

The observations from these numerical experiments are consistent with the analytical predictions of Bunger (2013) with respect to the critical role of viscosity and geometry on the propensity for multiple simultaneous HF growth. Pressure loss through the perforations has not yet been implemented in the simulator so its role, as predicted by Bunger et al. (In Press), cannot yet be tested through numerical experiments with this simulator.

The simulations do, however, provide insight to the importance of the end effects of the array. That is, the array is not strictly uniform as in the analytical model of Bunger (2013) and Bunger et al. (In Press), but instead the outer HFs experience a different stress state than the inner HFs because they have one side that is not subjected to any stress shadow. In the case of widely-spaced PKN HFs the impact is that the outer HFs grow a few percent more rapidly than the inner HFs; from the perspective of estimating overall behavior this is arguably a negligible impact. For the radial viscosity-dominated and the short PKN array the end effects apparently determine which HFs are favored by localization, but the basic prediction of Bunger (2013) of the presence and timing of localization is supported.

From the perspective of overall behavior, the only variation from the analytical predictions of Bunger (2013) is relatively subtle and is found in the radial toughness-dominated case. Bunger (2013) predicts immediate localization and Bunger et al. (In Press) show that this immediate localization can be mediated by perforation losses such that all HFs can grow to a radius around 0.2 times the spacing. However, the numerical model shows an early period where all HFs grow until the radius becomes 0.1-0.2 times the spacing in the absence of perforation losses. Furthermore, as pointed out by Bunger (2013), the analytical model cannot discern whether localization will favor growth of 1 or 2 HFs. The numerical experiments indicate localization to $2 \mathrm{HFs}$ rather than 1 . The dominant factor in this 2 way partitioning of fluid rather than strict localization to a single HF is probably driven by the small but finite contribution of viscous dissipation for the numerical simulations in the toughness dominated regime. This small contribution is ignored by the analytical model. However, including it in order to better predict the nature of localization to 1 versus 2 HFs generally and early-time localization in the radial toughness-dominated cases specifically should be relatively straight forward and will comprise a useful extension of these research efforts. 
a)
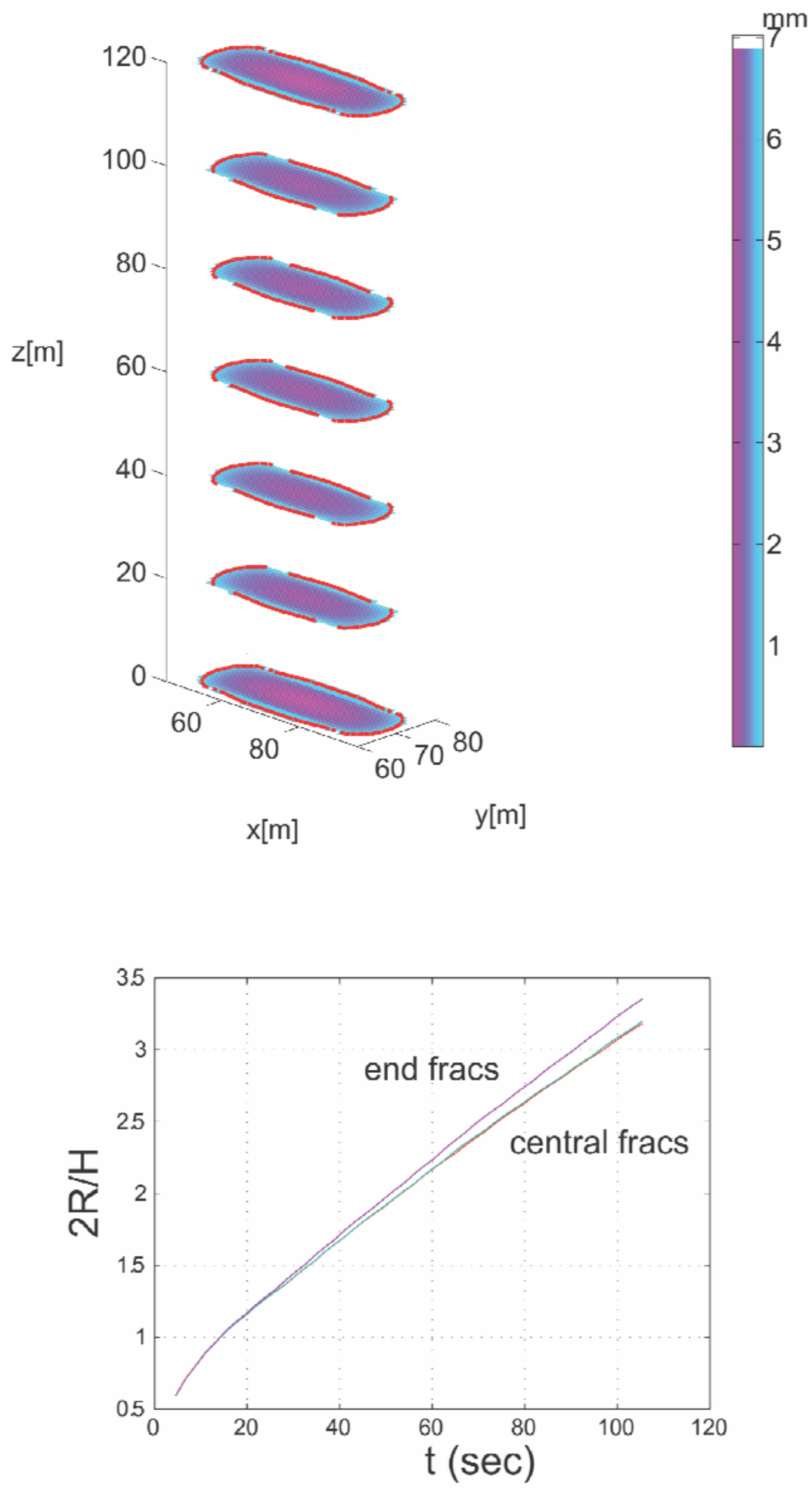

b)

FIG. 5. Height limited hydraulic fractures with spacing larger than the height. a) 7 hydraulic fractures with contours corresponding to opening. b) Evolution of the radius of the fractures normalized by the spacing between them. 


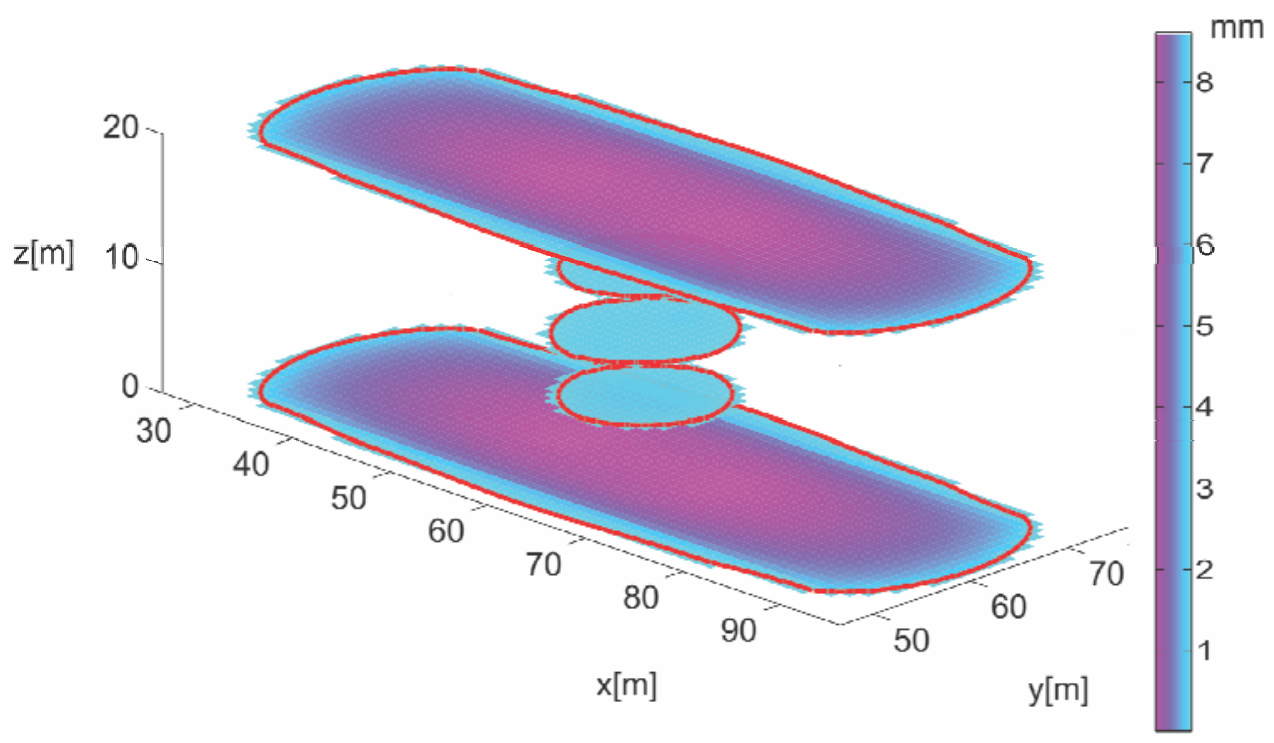

a)

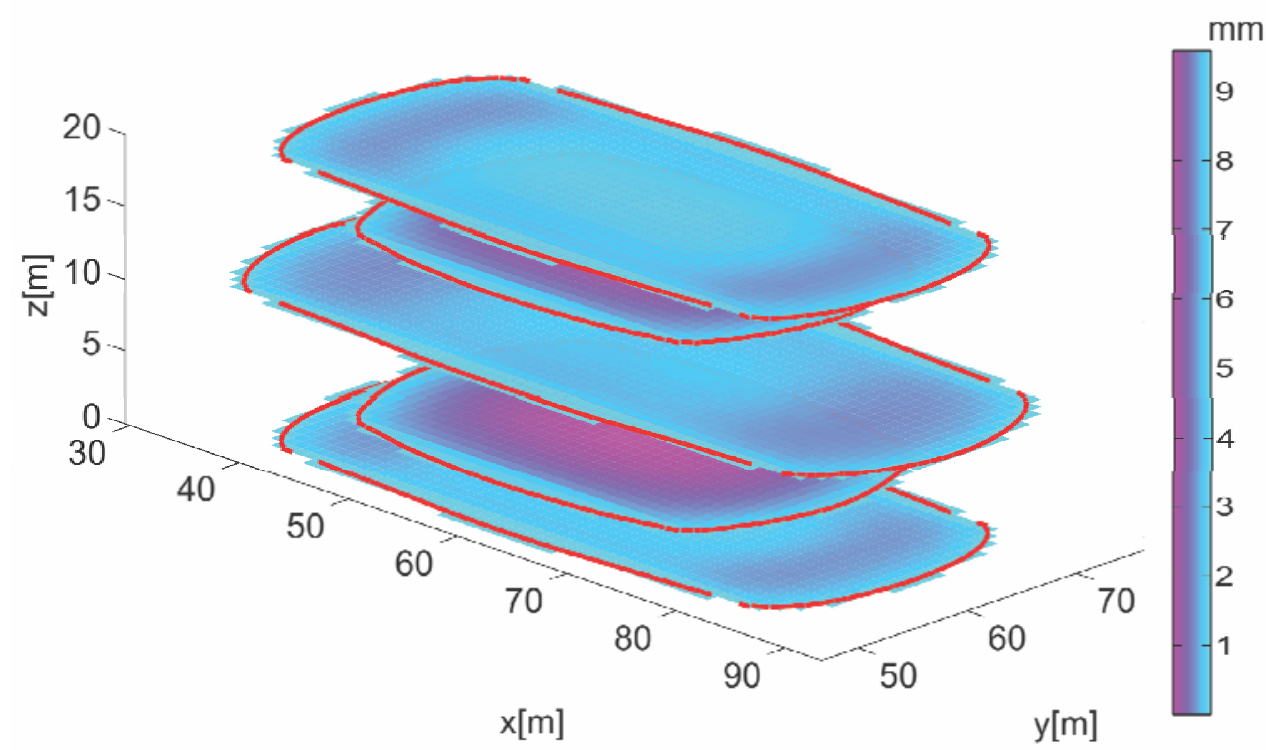

b)

FIG. 6. Height limited hydraulic fractures with spacing smaller than the height. a) Uniformly spaced HFs. b) Non-uniformly spaced HFs after Peirce and Bunger (In Review). 


\section{CONCLUSIONS}

The basic conditions that promote or suppress multiple simultaneous HF growth from arrays of entry points (i.e. perforation clusters) along horizontal wells are critical for design. Recent analytical models (Bunger 2013, Bunger et al. In press) are aimed at providing basic insights into this issue, while, on the other hand, design-oriented numerical models (e.g. Damjanac et al. 2010, Cipolla et al. 2011, Nagel et al. 2011, Meyer and Bazan 2011, Kresse et al. 2013, McClure and Horne 2013) are concerned with demonstrating details of the complexity that can characterize the problem. Here we present a new numerical model that aims at bridging the gap between theory and simulation. This fully-coupled, parallel planar 3D model has been used here to perform numerical experiments testing the predictions of Bunger (2013), namely that multiple simultaneous growth will be promoted by large viscous dissipation and height constraint. Both of these overall predictions of the theory are supported by the numerical experiments. Furthermore, we have observed some behaviors related to toughness-dominated HFs with small but finite viscous dissipation, as well as systems with 2 growing HFs, could be of practical importance and should be considered in future theoretical developments. Finally, we present an example showing that the complicated nature of systems of multiple HFs can lead to surprising results and unexpectedly simple strategies for promoting simultaneous growth that entail moderate perturbations to the locations of the perforation clusters.

\section{ACKNOWLEDGMENTS}

The authors appreciate the support of the International Engineering Foundation, the University of Pittsburgh Center for Energy, and the Natural Sciences and Engineering Research Council of Canada (NSERC).

\section{REFERENCES}

Abass, H. H., Soliman, M. Y., Tahini, A. M., Surjaatmadja, J., Meadows, D. L., and Sierra, L. (2009). Oriented fracturing: A new technique to hydraulically fracture an openhole horizontal well. Proceedings SPE Annual Technical Conference and Exhibition, New Orleans, LA, USA. SPE 124483.

Baihly, J. D., Malpani, R., Edwards, C., Han, S. Y., Kok, J. C. L., Tollefsen, E. M., and Wheeler, C. W. (2010). Unlocking the shale mystery: How lateral measurements and well placement impact completions and resultant production. Proceedings SPE Tight Gas Completions Conference, San Antonio, Texas, USA. SPE 138427.

Bunger, A. P. (2013). Analysis of the power input needed to propagate multiple hydraulic fractures. Int. J. Solids Struct., 50, 1538-1549.

Bunger, A. P., Zhang, X., and Jeffrey, R. G. (In Press). Constraints on simultaneous growth of hydraulic fractures from multiple perforation clusters in horizontal wells. Soc. Pet. Eng. J.

Cipolla, C., Weng, X., Onda, H., Nadaraja, T., Ganguly, U., and Malpani, R. (2011). New algorithms and integrated workflow for tight gas and shale completions. Proceedings SPE Annual Technology Conference and Exhibition, Denver, Colorado, USA. SPE 146872. 
Damjanac, B., Gil, I., Pierce, M., Sanchez, M., van As, A., and McLennan, J. (2010). A new approach to hydraulic fracturing modeling in naturally fractured reservoirs. Proceedings 44th U.S. Rock Mechanics Symposium, Salt Lake City, Utah, USA. ARMA 10-400.

Detournay, E. (2004). Propagation regimes of fluid-driven fractures in impermeable rocks. Int. J. Geomechanics, 4(1), 1-11.

Economides, M. and Nolte, K., eds. (2000). Reservoir Stimulation. John Wiley \& Sons, Chichester UK, 3rd ed.

Fisher, M. K., Heinze, J. R., Harris, C. D., Davidson, B. M., Wright, C. A., and Dunn, K. P. (2004). Optimizing horizontal completion techniques in the barnett shale using microseismic fracture mapping. Proceedings SPE Annual Technology Conference and Exhibition, Houston, Texas, USA. SPE 90051.

Germanovich, L. N., Ring, L. M., Astakhov, D. K., Shlyopobersky, J., and Mayerhofer, M. J. (1997). Hydraulic fracture with multiple segments II: Modeling. Int. J. Rock Mech. Min. Sci., 34(3-4), 472.

Howard, G. and Fast, C., eds. (1970). vol. 2. Henry L. Doherty Fund, SPE, New York.

King, G. E. (2010). Thirty years of gas shale fracturing: What have we learned? Proceedings SPE Annual Technical Conference and Exhibition, Florence, Italy. SPE 133256.

Kresse, O., Weng, X., Gu, H., and Wu, R. (2013). Numerical modeling of hydraulic fractures interaction in complex naturally fractured formations. Rock Mechanics and Rock Engineering, 46(3), 555-568.

Lecampion, B. and Detournay, E. (2007). An implicit algorithm for the propagation of a plane strain hydraulic fracture with fluid lag. Computer Meth. Appl. Mech. Eng, 196(49-52), 4863-4880.

McClure, M. W. and Horne, R. N. (2013). Discrete Fracture Network Modeling of Hydraulic Stimulation: Coupling Flow and Geomechanics. Springer Briefs in Earth Sciences. Springer, New York.

Meyer, B. and Bazan, L. (2011). A discrete fracture network model for hydraulically induced fractures-theory, parametric and case studies. Proceedings SPE Hydraulic Fracturing Technology Conference and Exhibition, The Woodlands, Texas, USA. SPE 140514.

Miller, C. and Waters, G. (2011). Evaluation of production log data from horizontal wells drilled in organic shales. Proceedings SPE North American Unconventional Gas Conference and Exhibition, The Woodlands, Texas, USA. SPE 144326.

Nagel, N., Gil, I., Sanchez-Nagel, M., and Damjanac, B. (2011). Simulating hydraulic fracturing in real fractured rocks - overcoming the limits of pseudo3D models. Proceedings SPE Hydraulic Fracturing Technology Conference and Exhibition, The Woodlands, Texas, USA. SPE 140480.

Nordgren, R. (1972). Propagation of vertical hydraulic fractures. J. Pet. Tech., 253, 306-314. (SPE 3009).

Olson, J. E. (2004). Predicting fracture swarms - the influence of subcritical crack growth and the crack-tip process zone on joint spacing in rock. The initiation, 
propagation, and arrest of joints and other fractures, , J. W. Cosgrove and T. Engelder, eds., Geological Society, London, vol. 231, 73-87.

Olson, J. E. and Dahi-Taleghani, A. (2009). Modeling simultaneous growth of multiple hydraulic fractures and their interaction with natural fractures. Proceedings SPE Hydraulic Fracturing Technology Conference and Exhibition, The Woodlands, Texas, USA. SPE 119739.

Peirce, A. and Detournay, E. (2008). An implicit level set method for modeling hydraulically driven fractures. Computer Meth. Appl. Mech. Eng, 197, 28582885.

Peirce, A. P. and Bunger, A. P. (In Review). Interference fracturing: Non-uniform distributions of perforation clusters that promote simultaneous growth of multiple hydraulic fractures. Soc. Pet. Eng. J. pre-print available at: http://hdl.handle.net/2429/45492.

Perkins, T. and Kern, L. (1961). Widths of hydraulic fractures. J. Pet. Tech., Trans. AIME, 222, 937-949. 